\title{
Peter und Gustav Hartz - zwei Reformer im Kampf gegen den Sozialstaat
}

Peter Hartz, früher Personalvorstand des größten Automobilkonzerns in Europa, Leiter der Kommission „Moderne Dienstleistungen am Arbeitsmarkt" und Miturheber der nach ihm benannten vier Gesetze (Hartz I-IV), ist wegen seiner Verwicklung in den sog. VW-Skandal um "Lustreisen" für Manager wie Betriebsräte vom Landgericht Braunschweig zu zwei Jahren Gefängnis auf Bewährung verurteilt worden. Hier geht es allerdings weder um ihn als Person noch um sein Konzept, das die Arbeitslosigkeit nicht verringert, aber die Armut erhöht hat, ${ }^{1}$ sondern um einen Namensvetter von Peter Hartz, der bisher weitgehend unbekannt, aufgrund seiner Rolle als geistiger Vorläufer aktueller und Pionier während der Weimarer Republik entwickelter "Reformpläne" jedoch sehr interessant ist.

\section{Christoph Butterwegge}

\section{Hartz in Weimar - ein historischer Rückblick}

Schon bevor die Weltwirtschaftskrise 1929/32 das Sozialsystem der Weimarer Republik bis ins Mark erschütterte, traten Kritiker des Wohlfahrtsstaates auf den Plan, die seine Leistungsfähigkeit in Zweifel zogen und - ganz ähnlich wie heute - statt öffentlicher Verantwortung für die Hilfesuchenden mehr Privatinitiative forderten. Einer davon hieß - man höre und staune - Gustav Hartz, gehörte der DNVP an und war 1924 für ein paar Monate Reichstagsabgeordneter. Im Dezember 1928 erschien sein Buch „Irrwege der deutschen Sozialpolitik und der Weg zur sozialen Freiheit", das Furore machte, häufig rezensiert und heftig kritisiert wurde.

In seinem Buch stellte Gustav Hartz viele Fragen, die heute neoliberalen Kritikern des Sozialstaates auf den Nägeln brennen, auch wenn er noch nicht dieselben Antworten (z.B. Einführung der Praxisgebühr) wie sie gab: „Geht man nicht bedenkenlos ein dutzendmal zum Arzt, wenn einmal genügte - nur weil es die Kasse bezahlt?“² Hartz sah überall „Faulenzer und Drückeberger" den Sozialstaat plündern, für die „kein denkender Arbeiter einen Pfennig Arbeitslosenbeiträge bezahlen" wolle. ${ }^{3}$ Überhaupt stelle der damals gerade erst geschaffene Versicherungszweig für die Lohnarbeiter „,kein gutes Geschäft" dar. ${ }^{4}$ Um „den Mißbrauch der ungerechten und unnötigen Inanspruchnahme" unterbinden zu können bzw. „asoziale Elemente“ (bei Hartz in Anführungszeichen) nicht mehr „auf allgemeine Unkosten reisen“ zu

Prof. Dr. Christoph Butterwegge leitet die Abteilung für Politikwissenschaft an der Universität zu Köln lassen, wollte Gustav Hartz die Hilfe auf Bedürftige konzentrieren, was er sozialdarwinistisch begründete: „Eine soziale Politik darf nicht mit der Sorge um die Kranken, Invaliden, Witwen, Waisen und Arbeitslosen die Förderung der Lebenstüchtigen, Leistungsfähigen und Arbeitenden vergessen."

Wortreich klagte Gustav Hartz über „die Bleigewichte des Bürokratismus“, kritisierte angeblich viel zu hohe Verwaltungskosten der Sozialversicherung und forderte eine Abkehr von dem Glauben, „daß der Staat alles selber machen muß." ${ }^{6}$ Hartz fühlte sich als mutiger Kritiker, der einer Front mächtiger Verteidiger des „jetzigen erstarrten Systems" gegenüberstand. ${ }^{7}$ Die damalige prekäre Situation der Sozialversicherung erschien ihm als Finanz-, mehr noch als Vertrauenskrise, welche alle Beteiligten erfasst habe. ${ }^{8}$ Einer der Lieblingsbegriffe, die Hartz ständig verwendete, hieß „Reform“. Manchmal sprach er allerdings von einer „Revolution“, um damit grundlegende Veränderungen des Systems zu kennzeichnen, die seiner Meinung nach unabdingbar waren.

Gustav Hartz tat, was damals eher ungewöhnlich war: Er fragte, welche Rendite die Zahlungen der Arbeitnehmer in die Sozialversicherung abwarfen und ob eine selbst angesparte, kapitalgedeckte Rente nicht mehr Gewinn verspreche. Außerdem machte sich Hartz - hiermit gleichfalls modern wirkend - für „eigenverantwortliche Selbsthilfe" der Arbeitnehmer stark. In den Vordergrund rückte Hartz die „Eigenverantwortung“, von der man heute wieder häufig spricht, um damit zu bemänteln, dass sich Ämter und Behörden immer mehr aus der Verantwortung stehlen, die ihnen das Sozialstaatsgebot des Grundgesetzes auferlegt. Die „staatliche Zwangsversicherung“ wollte Hartz abschaffen und ein System der priva- 
ten Vorsorge errichten, das auf Zwangssparen hinauslief. ${ }^{9}$ Hiervon versprach sich Hartz einen Mentalitätswandel, der die abhängig Beschäftigten mit dem bestehenden Wirtschaftssystem aussöhnen sollte: „Es erscheint mir fraglos, daß eine ganz andere Auffassung bei den Arbeitnehmern über den Wert des Kapitals und bezüglich der Verantwortung für seinen Verbrauch und seine Mehrung entstehen muß, wenn jeder das Wachsen seines Kapitals täglich bzw. wöchentlich vor Augen hat. " ${ }^{10}$ Hier liegt eine Hauptgemeinsamkeit zwischen Gustav und Peter Hartz: Beide wollten die Arbeiterschaft in das kapitalistische Gesellschaftssystem integrieren - sei es durch die Bildung von Eigentum, sei es durch relativ hohe Löhne für die Stammbelegschaften (VW-Haustarifvertrag) und eine bis zur Korruption reichende Privilegierung ihrer führenden Repräsentanten (Luxus- bzw. Lustreisen für einzelne Betriebsräte).

Klar war Gustav Hartz, dass Übergangsschwierigkeiten nicht zu vermeiden sein würden, was er aber in Kauf nehmen zu müssen glaubte - heute würde man in diesem Zusammenhang von „schmerzhaften Reformen“ sprechen und sie mit diesem Argument gerade als notwendige Radikalkur empfehlen. Die „grundstürzende Änderung“ benötige „eine gehörige Portion Mut beim Gesetzgeber", damit dieser nicht vor den vielen zu erwartenden „Wenns" und „Abers" zurückschrecke, ${ }^{11}$ befand Gustav Hartz. 75 Jahre später bildete „Mut zur Veränderung" das Motto der Agenda 2010. Warum es zwangsläufig zu „sozialen Härten“ komme, erläuterte Hartz anhand des folgenden Beispiels, das bei der Einführung der kapitalgedeckten Riester-Rente gleichfalls zu hören war: „Einem im vorgeschrittenen Alter von etwa 40 bis 50 Jahren stehenden Arbeitnehmer bleibt nicht mehr Zeit genug, um mit der Ersparung seiner Sozialbeiträge ein für Alter und Krankheit ausreichendes Sparkapital zusammenzubringen." ${ }^{12}$

An die Stelle der Sozialversicherung wollte Gustav Hartz „soziale Gemeinschaften“ (Gewerkschaften, Arbeitgeberverbände, Genossenschaften, Religionsgemeinschaften usw.) mit von ihnen betriebenen „Sozialsparkassen" treten lassen. Die einen Zankapfel der Politik bildenden Arbeitgeberbeiträge zur Sozialversicherung sollten dem Bruttolohn zugeschlagen, davon 15 Prozent als Sparbetrag abgeführt werden. Hartz hoffte, dies würde zur Folge haben, dass der ständige Streit über die „soziale Belastung der Wirtschaft" (heute hieße es: die Explosion der Lohnnebenkosten) unterbliebe. ${ }^{13}$ Unklar blieb, wie ein Wegfall der Arbeitgeberbeiträge angesichts des Machtvorsprungs der Unternehmer durch Lohn- und Gehaltssteigerungen kompensiert werden sollte. Auch würde sich der durch die Hinweise auf überhöhte, im Weltmaßstab nicht „konkurrenzfähige“ Lohnnebenkosten entfachte Druck des Kapitals nur andere Kanäle zur Entladung suchen. Statt der Personalzusatz- stünden vermutlich die Lohnkosten dann selbst noch stärker als bisher im Brennpunkt von Kampagnen.

Von den Erwerbslosen sprach Gustav Hartz - in gewisser Weise entsprechende Gedanken seines berühmten
Namensvetters vorwegnehmend - als „Kunden“ (in Anführungszeichen), die sich nach ihrer Entlassung „sofort bei der Arbeitsvermittlung zu melden“ hätten, damit diese sie kennen lerne und „die beste Kontrolle“ habe. ${ }^{14}$

Hartz wandte sich sowohl gegen Forderungen der Freien Gewerkschaften nach einer Verkürzung der Wochenarbeitszeit wie auch gegen (überhöhte) Sozialtransfers für Erwerbslose: „Es ist ein geradezu absurder Gedanke, der Arbeitslosigkeit mit Unterstützungsleistungen $\mathrm{zu}$ begegnen oder sie damit auch nur mildern zu wollen. Es ist ein absurder Gedanke, die noch vorhandene Arbeit durch Verkürzung der Arbeitszeit auf möglichst viele Menschen verteilen zu wollen, ein Gedanke, der sich würdig der vom Marxismus verbreiteten Meinung anschließt, dass der Einzelne möglichst wenig arbeite, damit er anderen die Arbeit nicht wegnähme." ${ }^{15}$ Stattdessen plädierte Hartz für Aufrüstung („Wiedergewinnung unserer Wehrhoheit") und eine allgemeine Arbeitsdienstpflicht der Jugend. Um der ihnen gesetzlich auferlegten Sparpflicht nachkommen und genügend Kapital im Rahmen der Sozialkassen ansparen zu können, sollten die Arbeitnehmer/innen laut Hartz täglich mindestens 9 Stunden im Büro oder Betrieb verbringen: „Eine Stunde Mehrarbeit am Tage, als Sparstunde genützt, würde weit sozialer wirken, als der Achtstundentag je an sozialer Wirkung zeitigen kann." 16

Wenn man den gegenwärtigen Demografie-Diskurs und das mit ihm verbundene Katastrophenszenario betrachtet, ${ }^{17}$ wirkt Gustav Hartz ebenfalls hochaktuell. In seinem als Fortsetzung der „Irrwege“ konzipierten Buch „Die national-soziale Revolution“, das gegen Ende des Jahres 1932 erschien, führte Gustav Hartz die Krise des von ihm „kollektivistisch“ genannten Sozialversicherungssystems auf demografische Veränderungen zurück. Immer mehr stark besetzte Jahrgänge rückten in die oberen Lebensaltersstufen ein, wie Bevölkerungswissenschaftler auch damals konstatierten: „Diese ,Vergreisung“ unseres Volkes hat eine dauernd steigende Zahl der Rentner und eine fortgesetzt steigende Rentenzahlungsdauer und absinkenden Beitragseingang im Gefolge." 18 Man erlebe gerade das Vorspiel einer Tragödie, meinte Hartz weiter: „In einer Reihe von Jahren sind nicht mehr genug junge beitragszahlende Menschen da, die in der Lage sind, die Summen aufzubringen, die zur Ernährung einer immer größer werdenden Zahl von Alten und Invaliden nötig werden. " ${ }^{19}$ Entweder müssten die Beiträge um nahezu das Doppelte steigen oder die Renten um etwa die Hälfte sinken. Als ein möglicher Ausweg erschien Hartz der systematische Aufbau individuell-familiärer Vorsorge, gekoppelt an die Pflicht zur Selbsthilfe und die persönliche Eigenverantwortung.

Zudem hielt Hartz eine „Höherbesteuerung der Ledigen und Kinderlosen" für sinnvoll, die zu fordern man nicht wage, weil „der Mut zu einer positiven Bevölkerungspolitik" fehle. ${ }^{20}$ Hans-Werner Sinn, Präsident des ifo Instituts für Wirtschaftsforschung an der Universität München, setzt heute gleichfalls auf eine aktive Bevölkerungspolitik 
zur Problembewältigung: „Wenn es gelänge, die Geburtenraten auf ein Niveau anzuheben, wie es eine stationäre Bevölkerung kennzeichnet, dann ließe sich die Bevölkerung allmählich wieder verjüngen. Das Rentenproblem würde sich lösen, der Arbeitsmarkt würde stabilisiert, und unser Land würde wieder zu der Dynamik bei der Wirtschaft und Wissenschaft zurückkehren, die es einmal besaß." ${ }^{21}$ Um dieses Ziel zu erreichen, will Sinn die Fertilitätsrate mittels finanzieller Anreize für Familien, aber auch mittels gezielter Sanktionen für Kinderlose steigern. Sinn empfiehlt die Staffelung von Altersrenten nach der Kinderzahl und eine Rentenkürzung für Kinderlose auf die Hälfte der „normalen“ Höhe: „Wer keine Kinder hat und insofern zu wenig tut, um seine eigene Rente im Umlagesystem zu sichern, muss die Konsequenzen tragen und selbst auf dem Wege der Ersparnis für Ersatz sorgen." ${ }^{22}$ Damals schrieb Gustav Hartz ähnlich klingende Sätze, die jedoch noch mehr Pathos enthielten: „Mit aller Deutlichkeit muß jedem zum Bewußtsein gebracht werden, daß die Zukunft unseres Volkes und Reiches eine ausreichende und gesunde Kinderschar und daß der Mensch immer noch das wertvollste ,Produkt' ist. Wer an der Zukunft unseres Volkes durch eigene Kinder keinen Anteil hat - oder haben kann -, der soll wenigstens die Gegenwart für die Kinderreichen materiell erträglich gestalten helfen. ${ }^{\text {23 }}$

Es mutet wie ein Treppenwitz der Geschichte an, dass die "Sozialstaatsreformer" damals und heute denselben Familiennamen hatten. Über bis zu der eher kuriosen Namensgleichheit von Hauptakteuren reichenden Gemeinsamkeiten dürfen wichtige Unterschiede zwischen Gegenwart und Vergangenheit jedoch nicht übersehen werden. Es gab in der Weimarer Republik nur einen ansatzweise entwickelten Wohlfahrtsstaat, was für die über 6 Millionen offiziell registrierten Erwerbslosen auf dem Höhepunkt der Weltwirtschaftskrise hieß, dass ihre Lage viel dramatischer war als die der Betroffenen heute. Sie hatten oft nichts zu essen und kein Dach über dem Kopf. Zudem war die Arbeitslosenquote mehr als doppelt so hoch und auch die Weltmarktdynamik längst nicht so ausgeprägt wie in der Gegenwart. Während die führenden Ökonomen ähnliche Rezepte zur Lösung der Beschäftigungskrise vertraten, unterschieden sich die Sozialstruktur und die gesellschaftlichen Kräfteverhältnisse deutlich von den aktuell bestehenden Machtkonstellationen. Auch waren die Großunternehmen der Zwischenkriegszeit noch nicht annähernd so stark in die Weltwirtschaft integriert wie transnationale Konzerne heute. Von der „Globalisierung“ und ihren Folgen für das System der sozialen Sicherung war daher noch keine Rede, wenngleich es Anfänge einer Internationalisierung der Kapitalmärkte gab und sich die negativen Folgen des "Schwarzen Freitags“ an der New Yorker Börse im Oktober 1929 fast überall bemerkbar machten.

\section{Weltwirtschaftskrise, Reformen des Sozialstaates und das Ende der Demokratie}

Während der Weltwirtschaftskrise 1929/33 zerbrach nicht nur der gesellschaftspolitische Basiskonsens zwischen den Klassen bzw. deren organisierter Interessenrepräsentanz, den Arbeitgeberverbänden und Freien Gewerkschaften, sondern im März 1930 auch die Große Koalition, deren beide Flügelparteien keine Einigung über den Weg zur finanziellen Konsolidierung der Arbeitslosenversicherung erzielten. Die unternehmernahe DVP bestand auf einer Kürzung von Leistungen, wohingegen die SPD-Fraktion im Unterschied zu ihrem Reichskanzler Hermann Müller nur eine Anhebung der Beiträge (damaliger Satz: 3,5 Prozent) unterstützte. In heutiger Diktion würde man sagen, dass die Beitragssatzstabilität bei den bürgerlichen Koalitionären absolute Priorität genoss, weil die Erhöhung der Lohnnebenkosten verhindert und die internationale Wettbewerbsfähigkeit des Wirtschaftsstandorts Deutschland nicht gefährdet werden sollte.

Nachdem maßgebliche Kräfte die Sozialdemokratie aus der Reichsregierung verdrängt hatten, war die Rechtsentwicklung des Weimarer Staates vorprogrammiert. In kürzer werdenden Abständen folgten der Regierung Müller immer weniger legitimierte (Präsidial-)Kabinette, die zwar kein Konzept zur Krisenbewältigung hatten, aber die sozialen Grundrechte der Arbeitnehmer/innen und Erwerbslosen mit rasantem Tempo beschnitten sowie den Wohlfahrtsstaat und die Demokratie demontierten. Man hat rückblickend den Eindruck, dass die Weimarer Republik und ihr Sozialsystem bewusst zugrunde gerichtet wurden, wobei die „Reform der Arbeitslosenversicherung" ganz oben auf der politischen Agenda stand. In weniger als 36 Monaten wurden die Leistungen der Arbeitslosenversicherung so stark beschnitten, die Anspruchsvoraussetzungen derart verschärft und die Bezugszeiten so massiv verkürzt, dass ganze Personengruppen (Jugendliche und verheiratete Frauen) überhaupt keinen Anspruch auf finanzielle Unterstützung mehr hatten und die übrigen höchstens noch 6 Wochen lang in deren Genuss kamen. „Das Versicherungsprinzip war somit am Ende der Weimarer Republik faktisch wieder abgeschafft."24

Unter dem christlichen Gewerkschafter und Zentrumspolitiker Heinrich Brüning, der vom 30. März 1930 bis zum 1. Juni 1932 Reichskanzler war, drang die Regierung auf mehr Zurückhaltung in der Lohnpolitik sowie bei den Staatsausgaben, wovon sie sich eine Sanierung des Budgets und eine Reaktivierung der Wirtschaft versprach. Letztere litt aber unter der fehlenden Nachfrage, die zu den nötigen Erweiterungsinvestitionen und damit neuen Arbeitsplätzen hätte führen können. Brüning hoffte auf die „Selbstheilungskräfte“ des Marktes, während das Geld für Konjunkturprogramme nach seiner Ansicht fehlte. Mit der Schwächung des Tarif- und Schlichtungswesens, dem Abbau der Arbeitslosenversicherung und der als „Sonderopfer des öffentlichen Dienstes“ deklarierten Senkung von Beamtengehältern und -pensionen begann 
unter Brüning ein Rückzug des Sozialstaates, der die Demokratie untergrub und den Weg zur NS-Diktatur ebnete. Eine für Exportbranchen vorteilhafte Deflationspolitik verschärfte die Wirtschafts- und Beschäftigungskrise.

Vorschläge, mit denen Unternehmerverbände und etablierte Politiker heute das System der sozialen Sicherung „verschlanken“ wollen, gleichen den damals diskutierten bzw. ergriffenen Maßnahmen teilweise bis ins Detail. Dies gilt z.B. für die Reform des Föderalismus, den Bürokratieabbau, die Schwächung des Tarifvertragssystems, die Erleichterung von Kündigungen und die Liberalisierung des Ladenschlusses, mit denen man die Wirtschaft bzw. ihre Konkurrenzfähigkeit auf dem Weltmarkt stärken wollte, genauso wie für Lohn- bzw. Gehaltssenkungen, unter denen die Binnenkaufkraft zu leiden hatte, und die Verlängerung der Wochenarbeitszeit.

Brünings nur ein halbes Jahr lang amtierender Nachfolger Franz von Papen stützte sich unverhohlen auf Vorschläge der (Schwer-)Industrie, die eine Entrechtung der abhängig Beschäftigten und eine Entmachtung ihrer Gewerkschaften anstrebte. Übereinstimmend erklärten beide Reichskanzler zwar, den Sozialstaat durch Reformen in der Substanz erhalten zu wollen, demontierten ihn aber Schritt um Schritt, was seiner weiteren Zerstörung eher Vorschub leistete. „Brüning und von Papen folgten der sich verstärkt politisch zu Wort meldenden neoklassischen Wirtschaftstheorie, wonach der Staat nur die Option habe, seine Ausgaben der immer restriktiveren Einnahmeseite anzupassen und dabei durch Steuerzurückhaltung gegenüber der Wirtschaft, geringe Löhne und Sozialabgaben die Angebotsbedingungen der Wirtschaft zu verbessern." 25

Die sozialpolitische Agonie der Weimarer Republik begann mit einer Kontroverse um die Sanierung der Arbeitslosenversicherung. Sowohl zwischen den gesellschaftlichen Interessengruppen bzw. den Verbänden von Unternehmern und Beschäftigten wie auch zwischen den Gebietskörperschaften war heftig umstritten, wem die enormen Kosten der Massenarbeitslosigkeit aufgebürdet werden sollten. Das deutsche Unterstützungssystem war dreigliedrig: Versicherte, die erwerbslos wurden, erhielten zunächst höchstens 26 Wochen lang Arbeitslosenhilfe (Hauptunterstützung) und Familienzuschläge für ihre engsten Angehörigen. Danach gab es im Falle der Bedürftigkeit gleichfalls 26, später sogar 52 Wochen lang Krisenfürsorge, bevor die allgemeine Wohlfahrt (der Gemeinden) einsprang. Während das Reich erwerbsfähige Arbeitslose unterstützte, die keine Versicherungsleistungen mehr erhielten, oblag den Gemeinden die Wohlfahrtsunterstützung, in deren Genuss nicht oder nur eingeschränkt Erwerbsfähige kamen. Man sprach von Hauptunterstützungsempfängern, Krisenfürsorgeempfängern und Wohlfahrtserwerbslosen. „Schon 1932 fiel etwa die Hälfte aller offiziell arbeitslos gemeldeten Arbeiter und Angestellten unter die Wohlfahrtserwerbslosen, also unter jene Gruppe, der es - örtlich variierend - am weitaus schlechtesten ging." 26
Innerhalb des dreigliedrigen Systems kam es zu Umschichtungen, die Finanzierungsschwierigkeiten entsprachen, aber auch unterschiedlichen Interessenlagen der Hauptakteure und Machtverschiebungen entsprangen, die nicht zuletzt der steigenden Arbeitslosigkeit geschuldet waren. Gab es anfangs sogar Bemühungen, die kommunale Erwerbslosen- in der staatlichen und teilweise aus Versicherungsmitteln finanzierten Krisenfürsorge aufgehen zu lassen, dominierten ungefähr seit dem Jahreswechsel 1930/31 Bestrebungen, die Krisenunterstützung mit der Wohlfahrtsfürsorge unter einheitlicher Verwaltung der Gemeinden zu verschmelzen. Am Ende des zuletzt genannten Jahres kursierten Pläne der Kommunen wie der Arbeitgeberverbände, alle Zweige des bestehenden Unterstützungssystems auf der Grundlage des (für die Erwerbslosen kargen und sie entrechtenden) Fürsorgeprinzips zusammenzulegen. Man führte zur Begründung die mangelnde Transparenz und die Schwerfälligkeit des gegliederten Systems sowie die Kostspieligkeit einer Versicherungslösung an.

Da die Arbeitslosen wegen der Wirtschaftskrise immer schneller (aus der staatlichen Krisenfürsorge) „ausgesteuert" wurden, stiegen die finanzielle Belastung und die Verschuldung der Kommunen seit 1929/30 ganz enorm. „Der Grundsatz, alle arbeitsfähigen Arbeitslosen von der gemeindlichen Fürsorge fernzuhalten, zerbrach (...) an der Finanzlage des Reichs. Damit wurde die Finanzkrise im Reichshaushalt zwangsläufig und unmittelbar in die Haushalte der Gemeinden hineingetragen." ${ }^{27}$ Sowohl die öffentliche Reformdebatte wie auch die Regierungspolitik verschoben sich immer stärker von einer Fusion der beiden Fürsorgesysteme auf dem (niedrigeren) Niveau der Wohlfahrtshilfe in Richtung einer Preisgabe des Versicherungsprinzips. Dezentralisierung und Kommunalisierung der Arbeitslosenunterstützung führten fast zwangsläufig zu einer sozialen Nivellierung nach unten, die damit meistenteils auch bezweckt war.

In der ersten Verordnung des Reichspräsidenten „zur Behebung finanzieller, wirtschaftlicher und sozialer Notstände", die einen Monat nach Auflösung des Parlaments am 26. Juli 1930 erlassen wurde, ergänzten sich Steuererhöhungen einerseits sowie Leistungskürzungen im Bereich der Arbeitslosen- und Krankenversicherung andererseits. Obwohl das zuständige Reichsarbeitsministerium noch einen weiteren Ausbau der Krankenversicherung vorgeschlagen hatte, dominierten „Sparbemühungen“, die eine Mehrbelastung der Versicherten durch eine Krankenscheingebühr und einen Arzneimittelbeitrag hervorbrachten. ${ }^{28}$ Eine weitere Notverordnung vom 1. Dezember 1930 beinhaltete u.a. eine Erhöhung der Tabaksteuer, während die Vermögen-, Grund- und Gewerbesteuer gesenkt wurden.

Kurzfristig wurde im Frühjahr 1931 eine meist nach ihrem Vorsitzenden, dem früheren langjährigen Arbeitsminister Heinrich Brauns benannte „Gutachterkommission zur Arbeitslosenfrage" gebildet. ${ }^{29}$ Ihre zehn Mitglieder, die innerhalb weniger Monate drei Berichte (zur Arbeitszeitfrage und zum sog. Doppelverdienertum, zur 
Arbeitsbeschaffung sowie zur Arbeitslosenhilfe) unterbreiteten, ohne damit viel Wirkung zu erzielen, kamen aus Verwaltung und Wissenschaft. Dies kann als weiteres Indiz dafür gelten, dass immer stärker expertokratisch statt demokratisch agiert und am Parlament vorbei regiert wurde. Statt die Vorlage sämtlicher Gutachten abzuwarten und die Abgeordneten damit zu befassen, brachte die Reichsregierung eine weitere Notverordnung „zur Sicherung von Wirtschaft und Finanzen“ auf den Weg, die am 5. Juni 1931, an eben jenem Tag, als die Sachverständigenkommission ihr letztes Einzelgutachten herausgab, erlassen wurde. Sie enthielt ein ganzes Paket drastischer Kürzungen bei der Arbeitslosenhilfe und der Krisenfürsorge, die Einführung der Bedürftigkeitsprüfung für Frauen und Jugendliche, eine Verlängerung der Wartezeiten sowie eine Begrenzung der Anrechnungszeiten für die Leistungsfestsetzung.

Nach dem Zusammenbruch der österreichischen Credit-Anstalt am 11. Mai 1931 geriet Deutschland in den Strudel einer internationalen Banken- und Währungskrise, die das Heer der Arbeitslosen überall weiter vergrößerte. Je weniger sie Herr der riesigen ökonomischen und sozialen Probleme waren, umso häufiger griffen die Präsidialregime zum Mittel der Notverordnung. Sukzessive setzten sie das (damals noch wenig entwickelte) System der sozialen Sicherung außer Kraft, kündigten allerdings bei jeder Reformmaßnahme an, nach diesem für Großteile der Bevölkerung schmerzhaften Eingriff werde die Wirtschaft wieder Tritt fassen. Ludwig Preller, der das Standardwerk zur Geschichte der Weimarer Sozialpolitik verfasste und die Konjunkturabhängigkeit solcher Aktivitäten des Staates in einer kapitalistischen Gesellschaft hervorhob, erklärte die damalige Demontage des Wohlfahrtsstaates in erster Linie mit dem dramatischen Beschäftigungsrückgang und einer daraus resultierenden Schwäche der Gewerkschaften im Kampf gegen die Großindustrie. „Wirtschaftsschläge wie der Bankenkrach von 1931, die Weltwirtschaftskrise und die ungeheuerliche Entwicklung der Arbeitslosigkeit mußten sich unter diesen Umständen katastrophal für die Weimarer Sozialpolitik auswirken, sie trafen schließlich nicht zuletzt über die Sozialpolitik den Nerv der Weimarer Demokratie.“"

Alfred Hugenberg, Medienzar und Führer der Deutschnationalen, schaltete im Februar 1932 mehrere Zeitungsannoncen mit der Aussage "Sozial ist, wer Arbeit schafft". In einer Rundfunkansprache zur Reichstagswahl sagte Hugenberg am 28. Juli 1932, Sozialismus sei mit Erwerbslosigkeit gleichbedeutend. Dagegen habe ein "gesunder Staat" auch „eine gesunde Wirtschaft", woraus Hugenberg schloss: „Derjenige ist wirklich und wahrhaft sozial, der Arbeit schafft. "31 Fast wörtlich fand dieser Satz über 70 Jahre später auf Betreiben der von Metallindustriellen finanzierten „Initiative Neue Soziale Marktwirtschaft" großen Anklang bei etablierten Politikern und Parteien. Beispielsweise stellte der nordrhein-westfälische CDUVorsitzende Jürgen Rüttgers seinen Landtagswahlkampf im Frühjahr 2005 unter das Motto „Sozial ist, was Arbeit schafft". Es handelt sich dabei im Grunde um eine Sklavenhalterideologie, die Arbeit aus ihren sozialen Bezügen herauslöst und verabsolutiert. Gar nicht mehr gestellt wird die Frage nach dem Sinngehalt von Lohnarbeit, den Arbeitsbedingungen und der Entlohnung, vom Anspruch der Stellenbewerber/innen auf Berufs- und Qualifikationsschutz ganz zu schweigen.

Am 14. Juni 1932 höhlte Reichskanzler von Papen das Unterstützungssystem für Arbeitslose weiter aus, ohne es allerdings formal abzuschaffen. Durch die „Verordnung des Reichspräsidenten über Maßnahmen zur Erhaltung der Arbeitslosenhilfe und der Sozialversicherung sowie zur Erleichterung der Wohlfahrtslasten der Gemeinden" wurden die Struktur (Einführung der Bedürftigkeitsprüfung in die Arbeitslosenversicherung) und das Leistungsniveau der drei Systeme noch mehr angeglichen. Papen bediente sich desselben Mittels wie sein Vorgänger, um den noch von diesem gegründeten Freiwilligen Arbeitsdienst (besonders für Jugendliche) auszuweiten, den Versicherungscharakter der Arbeitslosenversicherung de facto aufzuheben und die Selbstverwaltung der Reichsanstalt zu stutzen. Ludwig Preller sah darin die Verwirklichung des Kanzlerwunsches nach einer autoritären Führung: „Gedenkt man schließlich noch des Vorgehens Papens zur Lockerung der Tarifverträge, also auf Beseitigung eines entscheidenden Faktors der Weimarer Sozialpolitik, so wird ersichtlich, in wie hohem Maße sich Papen zum Vollstrecker der Bestrebungen der Schwerindustrie gegen das Wesen der Weimarer Sozialpolitik machte." ${ }^{32}$

Werner Abelshauser beschreibt, wie sich damals der soziale Abstieg einer durchschnittlichen Familie gestaltete: „Ein arbeitsloser Facharbeiter mit drei Angehörigen erhielt im Sommer 1932 nach einer Wartezeit von 14 Tagen wöchentlich rund 17.- RM und damit etwa $50 \mathrm{vH}$ seines zuvor verdienten Lohnes. Gegenüber dem 1929 erzielten Einkommen kam dies einem realen Kaufkraftverlust von etwa $60 \mathrm{vH}$ gleich. Nach 26 Wochen wurde der Arbeitslose aus der Versicherung , ausgesteuert ${ }^{\prime}$ und bezog in der Regel für 32 Wochen von der Krisenfürsorge eine noch geringere Unterstützung, bis er schließlich zum ,Fall' für die kommunale Erwerbslosenwohlfahrt wurde, deren Sätze sich an diejenigen der Armenfürsorge anlehnten. “33 Die (dem heutigen Arbeitslosengeld entsprechende) Arbeitslosenhilfe erhielten am Ende nicht einmal mehr 10 Prozent der registrierten Erwerbslosen, während die mit dem heutigen Arbeitslosengeld II vergleichbare, ursprünglich als Brücke zwischen Arbeitslosenhilfe und Armenfürsorge gedachte Krisenunterstützung das Niveau der Fürsorgeleistung nicht mehr überschreiten durfte. ${ }^{34}$

Damals wollte man die Wettbewerbsfähigkeit der deutschen Industrie durch eine „Sparpolitik“ bei den Löhnen und in den öffentlichen Haushalten wiederherstellen bzw. spürbar erhöhen. Als das nur noch halbdemokratische Regierungs- und Parteiensystem diese Aufgabe gegen Ende der Weimarer Republik trotz drastischer Beschneidung vieler Sozialleistungen nicht erfüllte, drängten einflussreiche Industrie- und Bankenkreise auf eine Kabinettsbeteiligung der NSDAP, die am 31. Juli 1932 zur 
stärksten Partei geworden war und ihren größten Wahlsieg gefeiert, bei der Novemberwahl desselben Jahres aber schon wieder Stimmen verloren hatte. Generalleutnant Kurt von Schleicher, der Reichspräsident Paul von Hindenburg sehr nahe und für eine „Querfrontstrategie“ (Bündnis aller im nationalkonservativen Sinne „wohlmeinenden“ Kräfte, unabhängig von deren Lagerzugehörigkeit) stand, setzte in seiner kurzen, nur vom 3. Dezember 1932 bis zum 30. Januar 1933 dauernden Amtszeit keine sozialpolitischen Akzente mehr.

Ohne die labilen ökonomischen, politischen und sozialen Verhältnisse am Ende der Weimarer mit den Arbeits- und Lebensbedingungen in der Bundesrepublik gleichzusetzen, kann man überraschende Parallelen, aber auch eine Kontinuität hinsichtlich des Kampfes mächtiger Interessengruppen gegen die angeblich zu hohen Sozialleistungen und den Wohlfahrtsstaat insgesamt feststellen. Die von Experten, Verbänden und Politikern unterbreiteten Vorschläge zur Überwindung der Beschäftigungskrise und zur Lösung der Finanzierungsprobleme des sozialen Sicherungssystems ähnelten sich gleichfalls. Als entscheidende Lehre aus der Geschichte bleibt zu beachten, dass der Sozialstaat nicht mit einem Schlag beseitigt, sondern Schritt für Schritt demontiert und dass mit ihm letztlich auch die Demokrati zugrunde gerichtet wird.

\section{Fußnoten}

1 Vgl. hierzu: Gabriele Gillen, Hartz IV. Eine Abrechnung, Reinbek bei Hamburg 2004; Agenturschluss (Hrsg.), Schwarzbuch Hartz IV. Sozialer Angriff und Widerstand - eine Zwischenbilanz, Berlin/Hamburg 2006; Christoph Butterwegge, Krise und Zukunft des Sozialstaates, 3. Aufl. Wiesbaden 2006, S. 184 ff.

2 Gustav Hartz, Irrwege der deutschen Sozialpolitik und der Weg zur sozialen Freiheit, Berlin 1928, S. 18

3 Siehe ebd., S. 80

4 Ebd., S. 81

5 Gustav Hartz, Neue Wege der Sozialpolitik, Langensalza 1929, S. 21

6 Siehe ders., Irrwege der deutschen Sozialpolitik und der Weg zur sozialen Freiheit, a.a.O., S. 141
7 Siehe ders. Gustav Hartz, Eigentum oder Rente? - Eine Auseinandersetzung mit meinen Kritikern über das Thema: Sozialversicherung oder Sozialsparkasse?, Berlin 1930, S. 8

8 Vgl. ebd., S. 13

9 Siehe Gustav Hartz, Irrwege der deutschen Sozialpolitik und der Weg zur sozialen Freiheit, a.a.O., S. 134

10 Ebd., S. 204

11 Siehe ebd., S. 193

12 Ebd., S. 194

13 Vgl. Gustav Hartz, Neue Wege in der Sozialpolitik, a.a.O., S. 25

14 Siehe ders., Irrwege der deutschen Sozialpolitik und der Weg zur sozialen Freiheit, a.a.O., S. 199

15 Ders., Die national-soziale Revolution, a.a.O., S. 194

16 Gustav Hartz, Irrwege der deutschen Sozialpolitik und der Weg zur sozialen Freiheit, a.a.O., S. 109

17 Vgl. hierzu: Christoph Butterwegge, Demographie als Ideologie? - Zur Diskussion über Bevölkerungs- und Sozialpolitik in Deutschland, in: Peter A. Berger/Heike Kahlert (Hrsg.), Der demographische Wandel. Chancen für die Neuordnung der Geschlechterverhältnisse, Frankfurt am Main/New York 2006, S. 53 ff.

18 Gustav Hartz, Die national-soziale Revolution, a.a.O., S. 148

19 Ebd.

20 Siehe Gustav Hartz, Irrwege der deutschen Sozialpolitik und der Weg zur sozialen Freiheit, a.a.O., S. 137 f. (Hervorh. im Original)

21 Hans-Werner Sinn, Ist Deutschland noch zu retten?, 4. Aufl. München 2003, S. 369 f.

22 Ebd., S. 393

23 Gustav Hartz, Irrwege der deutschen Sozialpolitik und der Weg zur sozialen Freiheit, a.a.O., S. 138

24 Werner Balsen u.a., Die neue Armut. Ausgrenzung von Arbeitslosen aus der Arbeitslosenunterstützung, Köln 1984, S. 26

25 Jürgen Boeckh/Ernst-Ulrich Huster/Benjamin Benz, Sozialpolitik in Deutschland. Eine systematische Einführung, Wiesbaden 2004, S. 77

26 Jürgen W. Falter, Hitlers Wähler, München 1991, S. 292

27 Ludwig Preller, Sozialpolitik in der Weimarer Republik, Stuttgart 1949, S. 421 (Hervorh. im Original)

28 Vgl. ebd., S. 472

29 Vgl. hierzu und zum Folgenden: Christian Berringer, Sozialpolitik in der Weltwirtschaftskrise. Die Arbeitslosenversicherungspolitik in Deutschland und Großbritannien im Vergleich 1928-1934, Berlin 1999, S. 240 f.

30 Ludwig Preller, Sozialpolitik in der Weimarer Republik, a.a.O., S. 512

31 Alfred Hugenberg, Rundfunkansprache zur Reichstagswahl am 31. Juli 1932, 28. Juli 1932, www.dhm.de/lemo/html/dokumente/hugenberg, 18.11.2005

32 Ludwig Preller, Sozialpolitik in der Weimarer Republik, a.a.O., S. 521

33 Werner Abelshauser, Macht oder ökonomisches Gesetz? Sozialpolitik und wirtschaftliche Wechsellagen vom Kaiserreich zur Bundesrepublik, in: Georg Vobruba (Hrsg.), Der wirtschaftliche Wert der Sozialpolitik, Berlin 1989, S. 19

34 Vgl. Diether Döring, Sozialstaat, Frankfurt am Main 2004, S. 27 